\title{
Double grazing bifurcations of the non-smooth railway wheelset systems
}

\author{
Pengcheng $\mathrm{Miao}^{a, b}$, Denghui $\mathrm{Li}^{c}$, Shan $\mathrm{Yin}^{d}$, \\ Jianhua Xie ${ }^{a}$, Celso Grebogi ${ }^{e}$, Yuan Yue ${ }^{a, b *}$ \\ ${ }^{a}$ School of Mechanics and Engineering, Southwest Jiaotong University, \\ Chengdu 610031, China \\ ${ }^{b}$ Applied Mechanics and Structure Safety Key Laboratory of Sichuan Province, \\ Southwest Jiaotong University,Chengdu 610031, China \\ ${ }^{c}$ School of Mathematics and Statistics, Hexi University, \\ Zhangye 734000, China \\ ${ }^{d}$ State Key Laboratory of Advanced Design and Manufacture for Vehicle Body, Hunan University, \\ Changsha, 410082, China \\ ${ }^{e}$ Institute for Complex Systems and Mathematical Biology King's College, University of Aberdeen, \\ Aberdeen AB24 3UE, United Kingdom
}

\begin{abstract}
There are numerous non-smooth factors in railway vehicle systems, such as flange impact, dry friction, creep force, and so on. Such non-smooth factors heavily affect the dynamical behavior of the railway systems. In this paper, we investigate and mathematically analyze the double grazing bifurcations of the railway wheelset systems with flange contact. Two types of models of flange impact are considered, one is a rigid impact model and the other is a soft impact model. First, we derive Poincaré maps near the grazing trajectory by the Poincaré-section discontinuity mapping (PDM) approach for the two impact models. Then, we analyze and compare the near grazing dynamics of the two models. It is shown that in the rigid impact model the stable periodic motion of the railway wheelset system translates into a chaotic motion after the gazing bifurcation, while in the soft impact model a pitchfork bifurcation occurs and the system tends to the chaotic state through a period doubling bifurcation. Our results also extend the applicability of the PDM of one constraint surface to that of two constraint surfaces for autonomous systems.
\end{abstract}

Keywords: Railway wheelset; Non-smooth; PDM; Double grazing bifurcations.

\section{Introduction}

As an important means of transportation, railway vehicles play an important role in a country's economy and in our way of life. For improvement in the speed of railway vehicles, it is necessary to consider many factors in the research of vehicle dynamics. The non-smooth dynamics, being at the core in railway wheelset systems, is a very important topic, which has been paid considerable attention by researchers [1].

\footnotetext{
${ }^{*}$ Corresponding author, E-mail: leyuan2003@sina.com
} 
In railway vehicle system, the non-smooth factors mainly come from suspension system and wheel-rail contact system, such as dry friction, flange impact, non-smooth creeps between rail and wheel, and so on [1]. The non-smooth factors induced abundant dynamic phenomena of the railway vehicle system. Huilgol [2] derived the lateral and yaw motion equations of a railway axle and investigated the dynamics of the system. Kaas-Petersen [3] investigated the dynamics of a railway bogie with flange contact, being the first to identify chaos in railway vehicle systems. Thereby, Meijaard et.al. [4] did, in fact, detect chaos in the railway wheelset system. Later, Knudsen et.al. [5] considered flange contact to analyze the dynamics of a railway wheelset system by path following routines. The results show that the system has both symmetric and asymmetric oscillations and chaotic motion. Then, True and his coworkers $[6,7]$ continue a numerical investigation of the dynamical behavior and the effect of parameters on the dynamics of the railway wheelset system that was started by Knudsen et.al. [5]. Ahmadian and Yang [8,9] performed a bifurcation analysis of railway wheelset and bogie systems with flange contact and non-smooth yaw dampers. It is shown that the yaw damping have a mixed effect on the critical speed and flange contact contribute significantly to the hunting behavior. True and Asmund [10] investigated lateral dynamics of a railway freight wagon wheelset with dry damping. They found that the action of dry friction completely changes the bifurcation diagram, and that the longitudinal component of the dry friction damping force destabilizes the wagon. Hoffmann $[11,12]$ considered the dry friction and leaf spring non-smoothness to investigate the dynamics of the European two-axle railway freight wagons with UIC standard suspension. Gao et. al.[13], based on the bifurcation and stability theory, investigated symmetric and asymmetric bifurcation behaviours of a bogie system with non-smooth wheel-rail contact relation. True et.al. [14] compared the resulting solutions that are found with three different strategies of handling the non-smoothness that occur in railway vehicle dynamics. As a side work, they tested several integrators, both explicit and implicit ones, and evaluated their performances and compared with respect to accuracy, and computation time.

Grazing impact arises in non-smooth systems when the flow is tangent to the boundary at zero velocity in phase space $[15,16]$. Nordmark [17] investigated the motion near the grazing impact of a single degree of freedom impact oscillators by periodically forced, introduced the concept of discontinuity mapping and constructed the normal form map for grazing bifurcation of the system for the first. Chin et al. [18, 19] studied the dynamics of the Nordmark map after one parameter unfolding in detail. They found that there exist complex phenomena of the mapping such as period adding cascade. The topological and statistical properties of chaotic attractors of the Normark map were studied in [20,21]. Fredriksson and Nordmark [22] extended the previous investigations [17] to many degrees of freedom systems and gave a stability criterion for a local attractor near the grazing point. di Bernardo et al. [23] extended the results presented in [17] to n-dimensional piecewise-smooth systems and derived normal form maps for grazing bifurcation. For additional literature about grazing bifurcation, see, e.g., [24, 25].

In spite of those research works, detailed investigation of grazing bifurcation in the railway vehicle systems is still missing. In particular, in most papers about grazing bifurcation in vibro-impact systems $[26,27,28]$, only one constraint and a drive by harmonic excitation were 
considered. For such systems, it is convenient to use a local zero-time discontinuous mapping (ZDM) approach to study the near grazing dynamics. However, the differential equations of lateral motion of vehicle systems are usually autonomous, and with bilateral symmetry constraints. Therefore, the existing literature results cannot be directly applied. In this work, we consider two-degree-of-freedom autonomous railway wheelset system with bilateral constraints. A local Poincaré-section discontinuity mapping (PDM) approach technique is used to investigate the grazing bifurcation of the system.

The remaining of this paper is organized in the following way. In Section 2, the dynamical model of the railway wheelset is described. In Section 3, we construct the Poincaré mapping of the dynamics near the grazing for two impact models of railway wheelset. In Section 4, numerical simulations are carried out to validate the theoretical analysis. In Section 5, we present the conclusions. In the Appendix, we describe the PDM calculation of the soft impact model.

\section{Dynamical model of the railway wheelset}

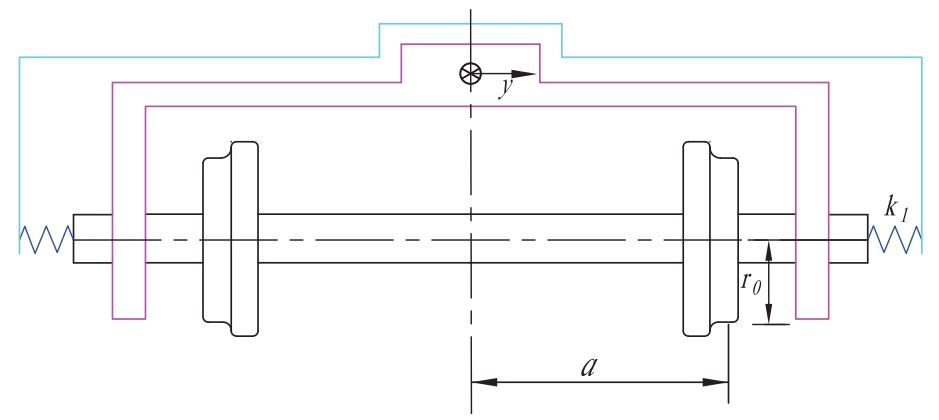

Figure 1: Model of railway wheelset and the coordinate system.

We consider the nonlinear dynamics of a single railway wheelset at a constant running speed on a straight track with flange. The wheels wheel profiles are conical and the conicity is $\lambda$. The railway wheelset is connected to the truck frame by a set of longitudinal and lateral springs, with no damping, as shown in Fig. 1. The railway wheelset has two degrees of freedom. Using the Newton-Euler method, the motion equations of the railway wheelset without flange impact are as follows $[5,6]$ :

$$
\begin{aligned}
m \ddot{y}+2 k_{1} y+2 F_{y} & =0, \\
I \ddot{\varphi}+2 k_{2} d_{1}^{2} \varphi+2 a F_{x} & =0,
\end{aligned}
$$

where $m$ is the mass of the wheelset, $I$ is the roll moment of inertia of the wheelset, $d_{1}$ is half of the primary yaw spring arm, $a$ is half of the track gauge, $r_{0}$ is the wheel radius, $k_{1}$ and $k_{2}$ are lateral stiffness and yaw spring stiffness of primary suspension, respectively, $F_{x}$ and $F_{y}$ are the longitudinal and lateral creep forces, respectively. 
The lateral and longitudinal creepages to the wheel and the rail are given by

$$
\left\{\begin{array}{c}
\xi_{y}=\frac{\dot{y}}{V}-\varphi \\
\xi_{x}=\frac{a \dot{\varphi}}{V}+\frac{\lambda y}{r_{0}} .
\end{array}\right.
$$

The nonlinear creep forces of the rail and wheels are calculated by Johnson and Vermeulen [29] contact theory. The creep force $F_{R}$ with lateral component $F_{y}$ and the longitudinal component $F_{x}$ are defined as

$$
\left\{\begin{array}{l}
F_{x}=\frac{\left(\frac{\xi_{x}}{\Phi}\right) F_{R}}{\xi_{R}} \\
F_{y}=\frac{\left(\frac{\xi_{y}}{\Psi}\right) F_{R}}{\xi_{R}}
\end{array}\right.
$$

where $\xi_{R}=\sqrt{\left(\frac{\xi_{x}}{\Phi}\right)^{2}+\left(\frac{\xi_{y}}{\Psi}\right)^{2}}$ is the resulting creepage, and the resulting creep force $F_{R}$ is expressed as

$$
F_{R}(u)=\mu N \begin{cases}u-\frac{1}{3} u^{2}+\frac{1}{27} u^{3}, & u<3 \\ 1, & u \geq 3\end{cases}
$$

where $u=\left(\frac{G \pi a_{e} b_{e}}{\mu N}\right) \xi_{R}$.

Now we introduce two models of flange impact,

\subsection{Soft impact model}

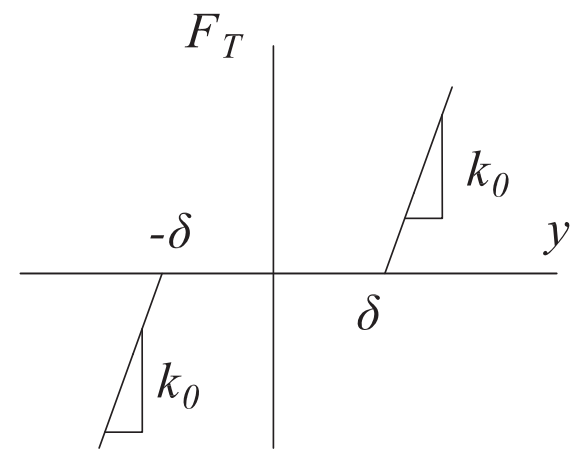

Figure 2: Flange contact force vs. lateral displacement.

The flange contact force $F_{T}(y)$ is approximated by a linear spring with deadband[5, 6], as shown in Fig. 2, which is described by

$$
F_{T}(y)=\left\{\begin{array}{lc}
k_{0}(y-\delta), & y>\delta \\
0, & -\delta \leq y \leq \delta \\
k_{0}(y+\delta), & y<-\delta
\end{array}\right.
$$

Then, the motion equations of the railway wheelset with flange impact are as follows $[5,6]$ :

$$
\begin{aligned}
m \ddot{y}+2 k_{1} y+2 F_{y}+F_{T}(y) & =0, \\
I \ddot{\varphi}+2 k_{2} d_{1}^{2} \varphi+2 a F_{x} & =0 .
\end{aligned}
$$


The dynamics of the system (2.6) has been investigated in detail by Knudsen [5, 6] and True[7], it is shown that there are abundant dynamical phenomena in the system (2.6), such as symmetry and asymmetry oscillations and chaos motion, but the detailed investigation of grazing bifurcation for the systems (2.6) is missing.

Let $y=x_{1}, \dot{y}=x_{2}, \varphi=x_{3}, \dot{\varphi}=x_{4}$. Then the equations (2.6) can be rewritten as the equivalent four-dimensional autonomous dynamical system as follows:

$$
\begin{aligned}
\dot{x_{1}} & =x_{2}, \\
\dot{x_{2}} & =-\frac{2 k_{1}}{m} x_{1}-\frac{2}{m} F_{y}-\frac{1}{m} F_{T}\left(x_{1}\right), \\
\dot{x_{3}} & =x_{4}, \\
\dot{x_{4}} & =-\frac{2 k_{2} d_{1}^{2}}{I} x_{3}-\frac{2 a}{I} F_{x},
\end{aligned}
$$

where $V$ is the velocity of the train, which is usually taken as a control parameter.

The systems (2.7) can be written in the general form:

$$
\dot{X}=\left\{\begin{array}{cc}
F_{2}(X, V), & H_{1}(X, V)>0, \\
F_{1}(X, V), & H_{1}(X, V) \leq 0 \leq H_{2}(X, V), \\
F_{3}(X, V), & H_{2}(X, V)<0,
\end{array}\right.
$$

where $X=\left(x_{1}, x_{2}, x_{3}, x_{4}\right)^{T}$ are the state variables of the system, and

$$
\begin{aligned}
& F_{2}(X, V)=\left(\begin{array}{c}
x_{2} \\
-\frac{2 k_{1}}{m} x_{1}-\frac{2}{m} F_{y}-\frac{k_{0}}{m}\left(x_{1}-\delta\right) \\
x_{4} \\
-\frac{2 k_{2} d_{1}^{2}}{I} x_{3}-\frac{2 a}{I} F_{x}
\end{array}\right), \\
& F_{1}(X, V)=\left(\begin{array}{c}
x_{2} \\
-\frac{2 k_{1}}{m} x_{1}-\frac{2}{m} F_{y} \\
x_{4} \\
-\frac{2 k_{2} d_{1}^{2}}{I} x_{3}-\frac{2 a}{I} F_{x}
\end{array}\right) \\
& F_{3}(X, V)=\left(\begin{array}{c}
x_{2} \\
-\frac{2 k_{1}}{m} x_{1}-\frac{2}{m} F_{y}-\frac{k_{0}}{m}\left(x_{1}+\delta\right) \\
x_{4} \\
-\frac{2 k_{2} d_{1}^{2}}{I} x_{3}-\frac{2 a}{I} F_{x}
\end{array}\right) .
\end{aligned}
$$

\section{$2.2 \quad$ Rigid impact model}

The flange contact can be considered as a rigid impact system. The equations governing the non-impact motions for $-\delta<y<\delta$ are given by equations (2.1):

$$
\begin{aligned}
m \ddot{y}+2 k_{1} y+2 F_{y} & =0, \\
I \ddot{\varphi}+2 k_{2} d_{1}^{2} \varphi+2 a F_{x} & =0 .
\end{aligned}
$$

When $y= \pm \delta$, the system satisfies the Newton's law of collisions, that is

$$
\dot{y}_{+}=-e \dot{y}_{-},
$$


where $0<e<1$ is the coefficient of restitution, and ' $+{ }^{\prime}$ represents the instantaneous moment after impact and ${ }^{\prime}{ }^{\prime}$ represents the instantaneous moment before impact.

Let $y=x_{1}, \dot{y}=x_{2}, \varphi=x_{3}, \dot{\varphi}=x_{4}$. Then the equations (2.1) can be rewritten as the equivalent four-dimensional autonomous dynamical system as follows:

$$
\begin{aligned}
\dot{x_{1}} & =x_{2}, \\
\dot{x_{2}} & =-\frac{2 k_{1}}{m} x_{1}-\frac{2}{m} F_{y} \\
\dot{x_{3}} & =x_{4}, \\
\dot{x_{4}} & =-\frac{2 k_{2} d_{1}^{2}}{I} x_{3}-\frac{2 a}{I} F_{x},
\end{aligned}
$$

The systems (2.10) can be written in the general form:

$$
\dot{X}=F_{1}(X, V),
$$

where $X=\left(x_{1}, x_{2}, x_{3}, x_{4}\right)^{T}$ are the state variables of the system, and

$$
F_{1}(X, V)=\left(\begin{array}{c}
x_{2} \\
-\frac{2 k_{1}}{m} x_{1}-\frac{2}{m} F_{y} \\
x_{4} \\
-\frac{2 k_{2} d_{1}^{2}}{I} x_{3}-\frac{2 a}{I} F_{x}
\end{array}\right) .
$$

\section{Grazing bifurcation of the railway wheelset}

Assume that there is a double grazing orbit, denoted by $\Gamma$, in the system (2.1), and that the grazing point of right constraint $\mathrm{A}$ is marked as $X_{1}^{*}$ with $X_{1}^{*}=\left(x_{1 A}^{*}, x_{2 A}^{*}, x_{3 A}^{*}, x_{4 A}^{*}\right)$. The impact surface $\Sigma_{1}$ of constraint $\mathrm{A}$ is defined as

$$
\Sigma_{1}=\left\{X \in D\left(X_{1}^{*}\right) \mid H_{1}(X, V)=x_{1}-\delta\right\} .
$$

Due to the existence of the symmetry, the grazing point of the left constraint B is marked as $X_{2}^{*}$ with $X_{2}^{*}=\left(x_{1 B}^{*}, x_{2 B}^{*}, x_{3 B}^{*}, x_{4 B}^{*}\right)$. The impact surface $\Sigma_{2}$ of constraint B is defined as

$$
\Sigma_{2}=\left\{X \in D\left(X_{2}^{*}\right) \mid H_{2}(X, V)=x_{1}+\delta\right\} .
$$

According to [16], the double grazing periodic motion satisfies the conditions

$$
\begin{aligned}
& H_{1}\left(X_{1}^{*}, V^{*}\right)=0, \\
& v_{1}\left(X_{1}^{*}, V^{*}\right)=\frac{\partial H_{1}}{\partial t}\left(\phi_{1}\left(X_{1}^{*}, V^{*}, 0\right), V^{*}\right)=H_{1, X} F_{1}\left(X_{1}^{*}, V^{*}\right)=0, \\
& a_{1}\left(X_{1}^{*}, V^{*}\right)=\frac{\partial^{2} H_{1}}{\partial^{2} t}\left(\phi_{1}\left(X_{1}^{*}, V^{*}, 0\right), V^{*}\right)=H_{1, X} F_{1, X} F_{1}\left(X_{1}^{*}, V^{*}\right)<0,
\end{aligned}
$$

and

$$
\begin{aligned}
& H_{2}\left(X_{2}^{*}, V^{*}\right)=0, \\
& v_{2}\left(X_{2}^{*}, V^{*}\right)=\frac{\partial H_{2}}{\partial t}\left(\phi_{1}\left(X_{2}^{*}, V^{*}, 0\right), V^{*}\right)=H_{2, X} F_{1}\left(X_{2}^{*}, V^{*}\right)=0, \\
& a_{2}\left(X_{2}^{*}, V^{*}\right)=\frac{\partial^{2} H_{2}}{\partial^{2} t}\left(\phi_{1}\left(X_{2}^{*}, V^{*}, 0\right), V^{*}\right)=H_{2, X} F_{1, X} F_{1}\left(X_{2}^{*}, V^{*}\right)>0 .
\end{aligned}
$$


We define two Poincaré surfaces

$$
\begin{gathered}
\Pi_{1}=\left\{X_{1} \in D_{1}\left(X_{1}^{*}\right) \mid v\left(X_{1}, V\right)=\frac{\partial H_{1}}{\partial t}\left(X_{1}, V\right)=H_{1, X} F_{1}\left(X_{1}, V\right)=0\right\}, \\
\Pi_{2}=\left\{X_{2} \in D_{2}\left(X_{2}^{*}\right) \mid v\left(X_{2}, V\right)=\frac{\partial H_{2}}{\partial t}\left(X_{2}, V\right)=H_{2, X} F_{1}\left(X_{2}, V\right)=0\right\},
\end{gathered}
$$

and two maps

$$
\begin{gathered}
P_{1}: \Pi_{1} \longmapsto \Pi_{2}, \\
P_{2}: \Pi_{2} \longmapsto \Pi_{1},
\end{gathered}
$$

where the flow maps are used to construct $P_{1}$ and $P_{2}$ governed by the vector field $F_{1}$ alone. That is, near the grazing points $X_{1}^{*}$ and $X_{2}^{*}$, we map points using only trajectories of the flow $\phi_{1}$ which generated by the vector field $F_{1}$, where $F_{1}$ represent the vector field without impact with the constraint. So the Poincaré maps $P_{1}$ and $P_{2}$ are defined by linearization

$$
\begin{gathered}
P_{1}(X, V)=X_{2}^{*}+N_{1}\left(X-X_{1}^{*}\right)+M_{1}\left(V-V^{*}\right)+\text { h.o.t }, \\
P_{2}(X, V)=X_{1}^{*}+N_{2}\left(X-X_{2}^{*}\right)+M_{1}\left(V-V^{*}\right)+\text { h.o.t, }
\end{gathered}
$$

respectively, where $N_{1}=\left.\frac{\partial P_{1}}{\partial X}\right|_{X=X_{1}^{*}, V=V^{*}}, M_{1}=\left.\frac{\partial P_{1}}{\partial V}\right|_{X=X_{1}^{*}, V=V^{*}}, N_{2}=\left.\frac{\partial P_{2}}{\partial X}\right|_{X=X_{2}^{*}, V=V^{*}}, M_{2}=$ $\left.\frac{\partial P_{2}}{\partial V}\right|_{X=X_{2}^{*}, V=V^{*} .} N_{1}$ and $N_{2}$ are $3 \times 3$ matrices, $M_{1}$ and $M_{2}$ are $3 \times 1$ matrices.

We next construct Poincaré maps in small neighborhoods of grazing points for the soft and the rigid railway wheelset impact models.

\subsection{The normal form map for soft impact model}

By Eqs. (5.16) of the Appendix A, the discontinuity mapping near grazing of the soft impact railway wheelset system can be defined as follows,

$$
P D M_{1}\left(X_{1}\right)= \begin{cases}X_{1}, & H_{1}\left(X_{1}, V\right)<0, \\ X_{1}+\beta_{1}\left(X_{1}-X_{1}^{*}\right) \sqrt{\frac{-2 H_{1}\left(X_{1}, V\right)}{a_{1}^{*}}}+ & \\ \beta_{2}\left(V-V^{*}\right) \sqrt{\frac{-2 H_{1}\left(X_{1}, V\right)}{a_{1}^{*}}+\text { h.o.t, }} & H_{1}\left(X_{1}, V\right) \geq 0,\end{cases}
$$

where $\beta_{1}=2\left(F_{2, X}^{*}-F_{1, X}^{*}\right)+\frac{2 F_{1}^{*}\left(a_{1, X}^{*}-a_{2, X}^{*}\right)}{a_{1}^{*}}, \beta_{2}=2\left(F_{2, V}^{*}-F_{1, V}^{*}\right)+\frac{2 F_{1}^{*}\left(a_{1, V}^{*}-a_{2, V}^{*}\right)}{a_{1}^{*}}$. The superscript $' *^{\prime}$ denotes quantities evaluated at the grazing point $\left(X^{*}, V^{*}\right)$, and 'h.o.t' represents the high order terms about $(X, V, t)$. We also define,

$$
P D M_{2}\left(X_{2}\right)= \begin{cases}X_{2}, & H_{2}\left(X_{2}, V\right)>0, \\ X_{2}+\beta_{3}\left(X_{2}-X_{2}^{*}\right) \sqrt{\frac{-2 H_{2}\left(X_{2}, V\right)}{a_{3}^{*}}}+ & \\ \beta_{4}\left(V-V^{*}\right) \sqrt{\frac{-2 H_{2}\left(X_{2}, V\right)}{a_{3}^{*}}+h . o . t,} & H_{2}\left(X_{2}, V\right) \leq 0,\end{cases}
$$

where $\beta_{3}=2\left(F_{3, X}^{*}-F_{1, X}^{*}\right)+\frac{2 F_{1}^{*}\left(a_{1, X}^{*}-a_{3, X}^{*}\right)}{a_{3}^{*}}, \beta_{4}=2\left(F_{3, V}^{*}-F_{1, V}^{*}\right)+\frac{2 F_{1}^{*}\left(a_{1, V}^{*}-a_{3, V}^{*}\right)}{a_{3}^{*}}$. Then the Poincaré map $P$ from $\Pi_{1}$ to itself can be written as

$$
P=P_{2} \circ P D M_{2} \circ P_{1} \circ P D M_{1}(X) .
$$


For $X_{1} \in \Pi_{1}$, we analyze the Poincaré map in the following way.

(1) For the case $H_{1}\left(X_{1}, V\right)<0$,

$$
\begin{aligned}
X_{2}=P_{1} \circ P D M_{1}\left(X_{1}\right) & =X_{2}^{*}+N_{1}\left(P D M_{1}\left(X_{1}\right)-X_{1}^{*}\right)+M_{1}\left(V-V^{*}\right)+\text { h.o.t } \\
& =X_{2}^{*}+N_{1}\left(X_{1}-X_{1}^{*}\right)+M_{1}\left(V-V^{*}\right)+\text { h.o.t. }
\end{aligned}
$$

(i) If $H_{2}\left(X_{2}, V\right)>0$, then

$$
\begin{aligned}
P_{2} \circ P D M_{2} \circ P_{1} \circ P D M_{1}\left(X_{1}\right) & =P_{2} \circ P D M_{2}\left(X_{2}\right) \\
& =X_{1}^{*}+N_{2}\left(X_{2}-X_{2}^{*}\right)+M_{2}\left(V-V^{*}\right)+\text { h.o.t. }
\end{aligned}
$$

(ii) If $H_{2}\left(X_{2}, V\right) \leq 0$, then

$$
\begin{aligned}
P_{2} \circ P D M_{2} \circ P_{1} \circ P D M_{1}\left(X_{1}\right) & =P_{2} \circ P D M_{2}\left(X_{2}\right) \\
& =X_{1}^{*}+N_{2}\left(X_{2}+\beta_{3}\left(X_{2}-X_{2}^{*}\right) \sqrt{\frac{-2 H_{2}\left(X_{2}, V\right)}{a_{3}^{*}}}\right. \\
& \left.+\beta_{4}\left(V-V^{*}\right) \sqrt{\frac{-2 H_{2}\left(X_{2}, V\right)}{a_{3}^{*}}}-X_{2}^{*}\right) \\
& +M_{2}\left(V-V^{*}\right)+\text { h.o.t. }
\end{aligned}
$$

(2) For the case $H_{1}\left(X_{1}, V\right) \geq 0$,

$$
\begin{aligned}
X_{2}=P_{1} \circ P D M_{1}\left(X_{1}\right) & =X_{2}^{*}+N_{1}\left(P D M_{1}\left(X_{1}\right)-X_{1}^{*}\right)+M_{1}\left(V-V^{*}\right)+h . o . t \\
& =X_{2}^{*}+N_{1}\left(X_{1}+\beta_{1}\left(X_{1}-X_{1}^{*}\right) \sqrt{\frac{-2 H_{1}\left(X_{1}, V\right)}{a_{1}^{*}}}\right. \\
& \left.+\beta_{2}\left(V-V^{*}\right) \sqrt{\frac{-2 H_{1}\left(X_{1}, V\right)}{a_{1}^{*}}}-X_{1}^{*}\right)+M_{1}\left(V-V^{*}\right)+\text { h.o.t. }
\end{aligned}
$$

(i) If $H_{2}\left(X_{2}, V\right)>0$, then

$$
\begin{aligned}
P_{2} \circ P D M_{2} \circ P_{1} \circ P D M_{1}\left(X_{1}\right) & =P_{2} \circ P D M_{2}\left(X_{2}\right) \\
& =X_{1}^{*}+N_{2}\left(X_{2}-X_{2}^{*}\right)+M_{2}\left(V-V^{*}\right)+\text { h.o.t. }
\end{aligned}
$$

(ii) If $H_{2}\left(X_{2}, V\right) \leq 0$, then

$$
\begin{aligned}
P_{2} \circ P D M_{2} \circ P_{1} \circ P D M_{1}\left(X_{1}\right)= & P_{2} \circ P D M_{2}\left(X_{2}\right) \\
= & X_{1}^{*}+N_{2}\left(X_{2}+\beta_{3}\left(X_{2}-X_{2}^{*}\right) \sqrt{\frac{-2 H_{2}\left(X_{2}, V\right)}{a_{3}^{*}}}\right. \\
& \left.+\beta_{4}\left(V-V^{*}\right) \sqrt{\frac{-2 H_{2}\left(X_{2}, V\right)}{a_{3}^{*}}}-X_{2}^{*}\right) \\
& +M_{2}\left(V-V^{*}\right)+\text { h.o.t. }
\end{aligned}
$$


According to (3.14-3.19), for every point $X_{1} \in D_{1}\left(X_{1}^{*}\right)$ on the Poincaré surface $\Pi_{1}$, we have

$$
\begin{aligned}
P\left(X_{1}\right)= & P_{2} \circ P D M_{2} \circ P_{1} \circ P D M_{1}\left(X_{1}\right) \\
= & \begin{cases}X_{1}^{*}+N_{2}\left(X_{2}-X_{2}^{*}\right)+M_{2}\left(V-V^{*}\right)+h . o . t, & H_{1}\left(X_{1}, V\right)<0, H_{2}\left(X_{2}, V\right)>0, \\
& X_{1}^{*}+N_{2}\left(X_{2}+\beta_{3}\left(X_{2}-X_{2}^{*}\right) \sqrt{\frac{-2 H_{2}\left(X_{2}, V\right)}{a_{3}^{*}}}\right. \\
+\beta_{4}\left(V-V^{*}\right) \sqrt{\left.\frac{-2 H_{2}\left(X_{2}, V\right)}{a_{3}^{*}}-X_{2}^{*}\right)} & H_{1}\left(X_{1}, V\right)<0, H_{2}\left(X_{2}, V\right) \leq 0, \\
+M_{2}\left(V-V^{*}\right)+h . o . t, & H_{1}\left(X_{1}, V\right) \geq 0, H_{2}\left(X_{2}, V\right)>0, \\
X_{1}^{*}+N_{2}\left(X_{2}-X_{2}^{*}\right)+M_{2}\left(V-V^{*}\right)+h . o . t, \\
X_{1}^{*}+N_{2}\left(X_{2}+\beta_{3}\left(X_{2}-X_{2}^{*}\right) \sqrt{\frac{-2 H_{2}\left(X_{2}, V\right)}{a_{3}^{*}}}\right. & \\
+\beta_{4}\left(V-V^{*}\right) \sqrt{\left.\frac{-2 H_{2}\left(X_{2}, V\right)}{a_{3}^{*}}-X_{2}^{*}\right)} & \\
+M_{2}\left(V-V^{*}\right)+h . o . t, & H_{1}\left(X_{1}, V\right) \geq 0, H_{2}\left(X_{2}, V\right) \leq 0 .\end{cases}
\end{aligned}
$$

\subsection{The normal form map for rigid impact model}

According to Ref.[16], the discontinuity mapping near grazing of the rigid impact railway wheelset system can be defined as follows:

$$
P D M_{1}\left(X_{1}\right)= \begin{cases}X_{1}, & H_{1}\left(X_{1}, V\right)<0, \\ X_{1}+\beta \sqrt{\frac{-2 H_{1}\left(X_{1}, V\right)}{a_{1}^{*}}}+\text { h.o.t, } & H_{1}\left(X_{1}, V\right) \geq 0,\end{cases}
$$

where $\beta_{5}=-\left(W a_{1}^{*}-\left(v_{1 X}^{*} W\right) F_{1}\left(X_{1}^{*}, V^{*}\right)\right), v_{1 X}^{*}=\left.\left(H_{X} F_{1}\right)_{X}\right|_{X=X_{1}^{*}, V=V^{*}}, v_{1 X}^{*} W=-(1+e), W=$ $(0,0,0)^{T}$, Furthermore,

$$
P D M_{2}\left(X_{2}\right)= \begin{cases}X_{2}, & H_{2}\left(X_{2}, V\right)>0, \\ X_{2}+\beta_{6} \sqrt{\frac{-2 H_{2}\left(X_{2}, V\right)}{a_{2}^{*}}}+\text { h.o.t, } & H_{2}\left(X_{2}, V\right) \leq 0,\end{cases}
$$

where $\beta_{6}=-\left(W a_{2}^{*}-v_{2 X}^{*} W F_{1}\left(X_{2}^{*}, V^{*}\right)\right)$ and $v_{2 X}^{*}=\left.\left(H_{X} F_{1}\right)_{X}\right|_{X=X_{2}^{*}, V=V^{*}}$.

Then the Poincré map $P$ from $\Pi_{1}$ to itself can be written as

$$
P(X)=P_{2} \circ P D M_{2} \circ P_{1} \circ P D M_{1}(X) .
$$

For $X_{1} \in \Pi_{1}$, we analyze the Poincaré map in the following way.

(1) For the case $H_{1}\left(X_{1}, V\right)<0$,

$$
\begin{aligned}
X_{2}=P_{1} \circ P D M_{1}\left(X_{1}\right) & =X_{2}^{*}+N_{1}\left(P D M_{1}\left(X_{1}\right)-X_{1}^{*}\right)+M_{1}\left(V-V^{*}\right)+\text { h.o.t } \\
& =X_{2}^{*}+N_{1}\left(X_{1}-X_{1}^{*}\right)+M_{1}\left(V-V^{*}\right)+\text { h.o.t. }
\end{aligned}
$$

(i) If $H_{2}\left(X_{2}, V\right)>0$, then

$$
\begin{aligned}
P_{2} \circ P D M_{2} \circ P_{1} \circ P D M_{1}\left(X_{1}\right) & =P_{2} \circ P D M_{2}\left(X_{2}\right) \\
& =X_{1}^{*}+N_{2}\left(X_{2}-X_{2}^{*}\right)+M_{2}\left(V-V^{*}\right)+\text { h.o.t. }
\end{aligned}
$$


(ii) If $H_{2}\left(X_{2}, V\right) \leq 0$, then

$$
\begin{aligned}
P_{2} \circ P D M_{2} \circ P_{1} \circ P D M_{1}\left(X_{1}\right)= & P_{2} \circ P D M_{2}\left(X_{2}\right) \\
= & X_{1}^{*}+N_{2}\left(X_{2}+\beta \sqrt{\frac{-2 H_{2}\left(X_{2}, V\right)}{a_{2}^{*}}}-X_{2}^{*}\right) \\
& +M_{2}\left(V-V^{*}\right)+\text { h.o.t. }
\end{aligned}
$$

(2) For the case $H_{1}\left(X_{1}, V\right) \geq 0$,

$$
\begin{aligned}
X_{2}=P_{1} \circ P D M_{1}\left(X_{1}\right) & =X_{2}^{*}+N_{1}\left(P D M_{1}\left(X_{1}\right)-X_{1}^{*}\right)+M_{1}\left(V-V^{*}\right)+\text { h.o.t } \\
& =X_{2}^{*}+N_{1}\left(X_{1}+\beta_{5} \sqrt{\frac{-2 H_{1}\left(X_{1}, V\right)}{a_{1}^{*}}}-X_{1}^{*}\right)+M_{1}\left(V-V^{*}\right)+\text { h.o.t. }
\end{aligned}
$$

(i) If $H_{2}\left(X_{2}, V\right)>0$, then

$$
\begin{aligned}
P_{2} \circ P D M_{2} \circ P_{1} \circ P D M_{1}\left(X_{1}\right) & =P_{2} \circ P D M_{2}\left(X_{2}\right) \\
& =X_{1}^{*}+N_{2}\left(X_{2}-X_{2}^{*}\right)+M_{2}\left(V-V^{*}\right)+\text { h.o.t. }
\end{aligned}
$$

(ii) If $H_{2}\left(X_{2}, V\right) \leq 0$, then

$$
\begin{aligned}
P_{2} \circ P D M_{2} \circ P_{1} \circ P D M_{1}\left(X_{1}\right)= & P_{2} \circ P D M_{2}\left(X_{2}\right) \\
= & X_{1}^{*}+N_{2}\left(X_{2}+\beta \sqrt{\frac{-2 H_{2}\left(X_{2}, V\right)}{a_{2}^{*}}}-X_{2}^{*}\right) \\
& +M_{2}\left(V-V^{*}\right)+\text { h.o.t. }
\end{aligned}
$$

According to (3.24-3.29), for every point $X_{1} \in D_{1}\left(X_{1}^{*}\right)$ on the Poincaré surface $\Pi_{1}$, we have

$$
\begin{aligned}
P\left(X_{1}\right)= & P_{2} \circ P D M_{2} \circ P_{1} \circ P D M_{1}\left(X_{1}\right) \\
= & \begin{cases}X_{1}^{*}+N_{2}\left(X_{2}-X_{2}^{*}\right)+M_{2}\left(V-V^{*}\right)+h . o . t, & H_{1}\left(X_{1}, V\right)<0, H_{2}\left(X_{2}, V\right)>0, \\
X_{1}^{*}+N_{2}\left(X_{2}+\beta 6 \sqrt{\frac{-2 H_{2}\left(X_{2}, V\right)}{a_{2}^{*}}}-X_{2}^{*}\right) & H_{1}\left(X_{1}, V\right)<0, H_{2}\left(X_{2}, V\right) \leq 0, \\
+M_{2}\left(V-V^{*}\right)+h . o . t, & (3.30) \\
X_{1}^{*}+N_{2}\left(X_{2}-X_{2}^{*}\right)+M_{2}\left(V-V^{*}\right)+h . o . t, & H_{1}\left(X_{1}, V\right) \geq 0, H_{2}\left(X_{2}, V\right)>0, \\
X_{1}^{*}+N_{2}\left(X_{2}+\beta_{6} \sqrt{\frac{-2 H_{2}\left(X_{2}, V\right)}{a_{2}^{*}}}-X_{2}^{*}\right) & \\
+M_{2}\left(V-V^{*}\right)+h . o . t, & H_{1}\left(X_{1}, V\right) \geq 0, H_{2}\left(X_{2}, V\right) \leq 0 .\end{cases}
\end{aligned}
$$

\section{Numerical simulations}

In this Section, we apply the discontinuity mapping approach to derive explicit expressions for the composite Poincaré map $P\left(X_{1}, V\right)$ in the neighborhood of selected grazing periodic orbit. 
Here the shooting method is used to obtain grazing periodic orbit. The corresponding parameters values are taken from Table 1 . The grazing points of periodic trajectory $\Gamma$ are

$$
\begin{aligned}
& X_{1}^{*} \approx(0.009100,0,0.000811,-0.013250)^{T}, \\
& X_{2}^{*} \approx(-0.009100,0,-0.000811,0.013250)^{T}, \\
& V^{*} \approx 10.056027 \mathrm{~m} / \mathrm{s} .
\end{aligned}
$$

Figure 3 is the phase portrait of the double grazing periodic orbit $\Gamma$. The blow up in the neighborhood of grazing points of Fig. 3 are shown in Fig. 4. Figure 5 is the time history of the displacement for the grazing periodic orbit $\Gamma$.

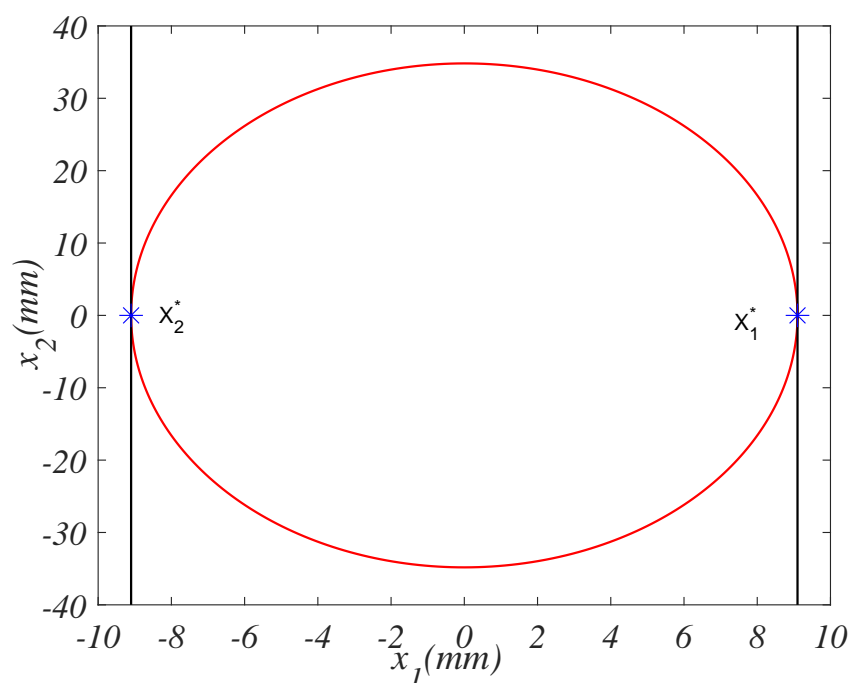

Figure 3: Phase portrait of the grazing periodic orbit in the $\left(x_{1}, x_{2}\right)$ plane.

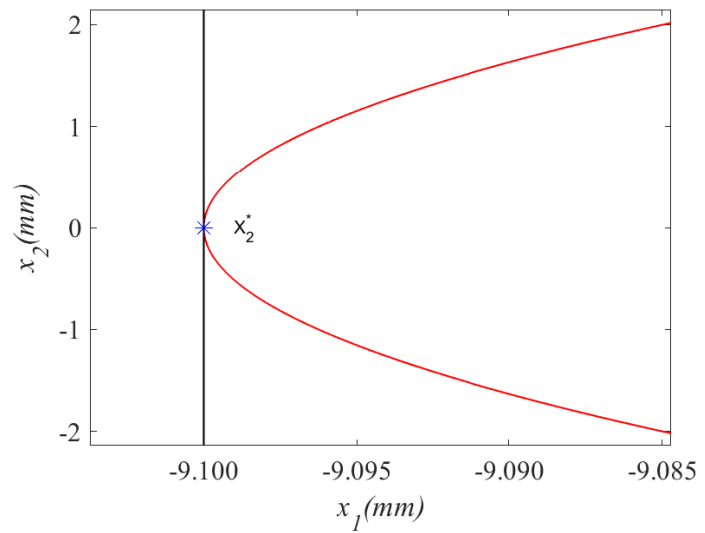

(a)

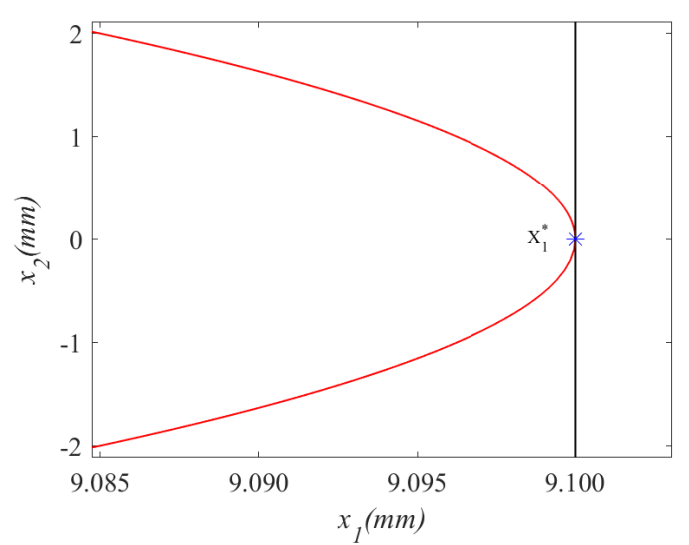

(b)

Figure 4: Blow up of phortraits in Fig. 3 near grazing points $X_{1}^{*}$ and $X_{2}^{*}$. 


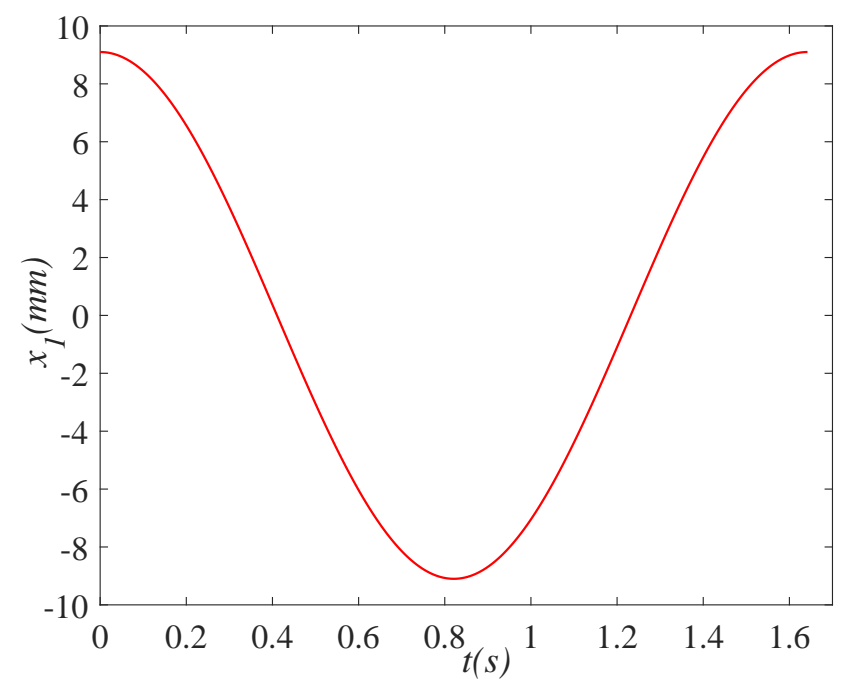

Figure 5: Time history of the displacement of the grazing periodic orbit.

For soft impact, the composite Poincaré map is

$$
P= \begin{cases}X_{1}^{* *}+\gamma_{1} \Delta X+\gamma_{2} \Delta V+\text { h.o.t, } & H_{1}\left(X_{1}, V\right)<0, H_{2}\left(X_{2}, V\right)>0, \\ X_{1}^{* *}+\gamma_{1} \Delta X+\gamma_{2} \Delta V+ & \\ \left(\gamma_{3} \Delta X+\gamma_{4} \Delta V\right) \sqrt{\gamma_{5} \Delta X+0.000856 \Delta V^{1}}+\text { h.o.t, } & H_{1}\left(X_{1}, V\right)<0, H_{2}\left(X_{2}, V\right) \leq 0, \\ X_{1}^{* *}+\gamma_{1} \Delta X+\gamma_{2} \Delta V+\gamma_{6} \Delta X \sqrt{\Delta X^{1}}+\text { h.o.t, } & H_{1}\left(X_{1}, V\right) \geq 0, H_{2}\left(X_{2}, V\right)>0,(4.1) \\ X_{1}^{* *}+\gamma_{1} \Delta X+\gamma_{2} \Delta V+\gamma_{6} \Delta X \sqrt{\Delta X^{1}} & \\ +\left(\gamma_{3} \Delta X+\gamma_{4} \Delta V+\gamma_{7} \Delta X \sqrt{\Delta X^{1}}\right) \times & \\ \sqrt{\gamma_{5} \Delta X+0.000856 \Delta V^{1}+\gamma_{6} \Delta X \sqrt{\Delta X^{1}}}+\text { h.o.t, } & H_{1}\left(X_{1}, V\right) \geq 0, H_{2}\left(X_{2}, V\right) \leq 0,\end{cases}
$$

where $\Delta X=X_{1}-X_{1}^{* *}, \Delta V=V-V^{*}, \Delta X^{1}$ is the first component of $\Delta X, \Delta V^{1}$ is the first component of $\Delta V$, and

$$
\begin{gathered}
X_{1}^{* *}=\left(\begin{array}{c}
0.009100 \\
0.000810 \\
-0.013249
\end{array}\right), \gamma_{1}=\left(\begin{array}{ccc}
0.952259 & -0.351723 & -0.053662 \\
0.085117 & -0.031439 & -0.004797 \\
-1.38605 & 0.511948 & 0.078108
\end{array}\right), \gamma_{2}=\left(\begin{array}{c}
0.001804 \\
0.000065 \\
-0.003700
\end{array}\right), \\
\gamma_{3}=\left(\begin{array}{ccc}
-4195.16 & 1549.51 & 236.409 \\
-374.980 & 138.501 & 21.1311 \\
6106.24 & -2255.38 & -344.103
\end{array}\right), \gamma_{4}=\left(\begin{array}{c}
-3.77022 \\
-0.336997 \\
5.48771
\end{array}\right), \gamma_{5}=\left(\begin{array}{c}
0.952770 \\
-0.351912 \\
-0.053691
\end{array}\right), \\
\gamma_{6}=\left(\begin{array}{ccc}
-4400.76 & 0 & 0 \\
-393.359 & 0 & 0 \\
6405.50 & 0 & 0
\end{array}\right), \gamma_{7}=\left(\begin{array}{ccc}
1.93875 e 7 & 0 & 0 \\
1.73293 e 6 & 0 & 0 \\
-2.82193 e 7 & 0 & 0
\end{array}\right) .
\end{gathered}
$$

Figure 6 shows the bifurcation diagrams of the system: (a) is obtained by direct numerical simulation and (b) is obtained by the local mapping for the soft impact model. It is shown that the qualitative properties of the two cases are almost the same, i.e., after the grazing bifurcation it has a pitchfork bifurcation and translates to chaos through period doubling. 


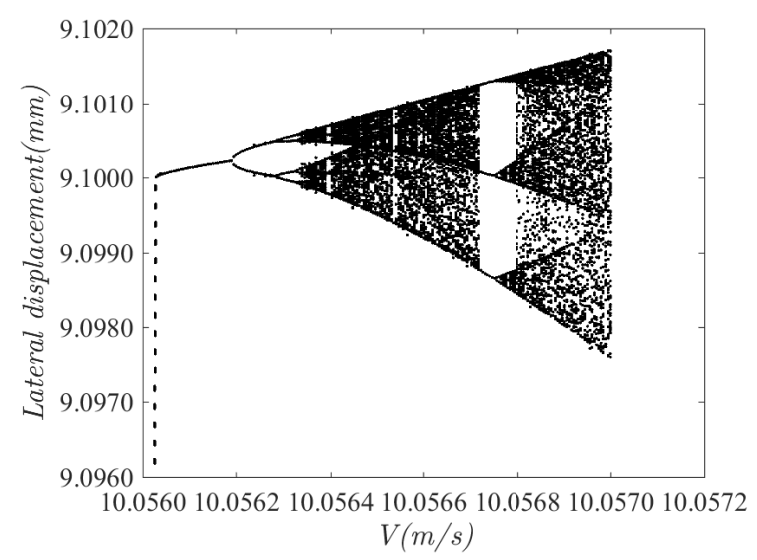

(a) Numerical simulation,

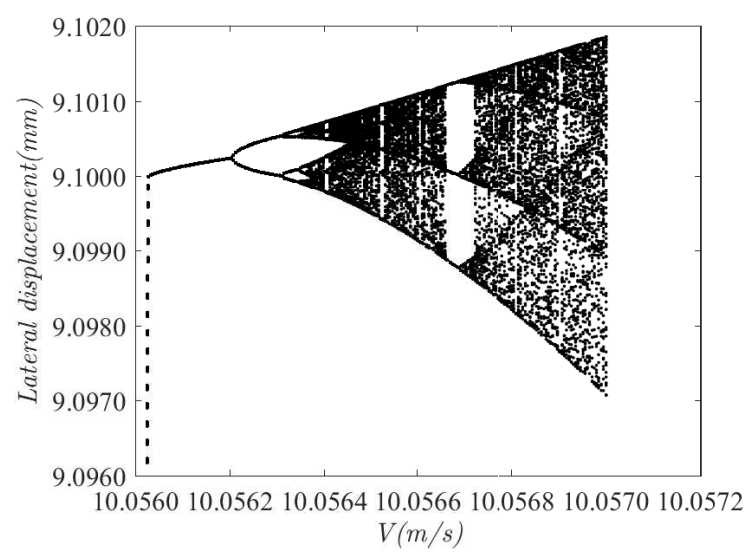

(b) The PDM

Figure 6: Bifurcation diagrams for the soft impact model.

For rigid impact, the composite Poincaré map is

$$
P= \begin{cases}X_{1}^{* *}+\gamma_{1}^{\prime} \Delta X+\gamma_{2}^{\prime} \Delta V+\text { h.o.t, } & H_{1}\left(X_{1}, V\right)<0, H_{2}\left(X_{2}, V\right)>0, \\ X_{1}^{* *}+\gamma_{1}^{\prime} \Delta X+\gamma_{2}^{\prime} \Delta V+\gamma_{3}^{\prime} \sqrt{\gamma_{4}^{\prime} \Delta X+0.000856 \Delta V^{1}}+\text { h.o.t, }, & H_{1}\left(X_{1}, V\right)<0, H_{2}\left(X_{2}, V\right) \leq 0, \\ X_{1}^{* *}+\gamma_{1}^{\prime} \Delta X+\gamma_{2}^{\prime} \Delta V+\gamma_{5}^{\prime} \sqrt{\Delta X^{1}}+\text { h.o.t, } & H_{1}\left(X_{1}, V\right) \geq 0, H_{2}\left(X_{2}, V\right)>0,(4.2) \\ X_{1}^{* *}+\gamma_{1}^{\prime} \Delta X+\gamma_{2}^{\prime} \Delta V+\gamma_{5}^{\prime} \sqrt{\Delta X^{1}}+ & \\ \gamma_{3}^{\prime} \sqrt{\gamma_{4}^{\prime} \Delta X+0.000856 \Delta V^{1}+0.036963 \sqrt{\Delta X^{1}}}+\text { h.o.t, } & H_{1}\left(X_{1}, V\right) \geq 0, H_{2}\left(X_{2}, V\right) \leq 0,\end{cases}
$$

where

$$
\begin{gathered}
X_{1}^{* *}=\left(\begin{array}{c}
0.009100 \\
0.000810 \\
-0.013249
\end{array}\right), \gamma_{1}^{\prime}=\left(\begin{array}{ccc}
0.952259 & -0.351723 & -0.053662 \\
0.085117 & -0.031439 & -0.004797 \\
-1.38605 & 0.511948 & 0.078108
\end{array}\right), \gamma_{2}^{\prime}=\left(\begin{array}{c}
0.001804 \\
0.000065 \\
-0.003700
\end{array}\right), \\
\gamma_{3}^{\prime}=\left(\begin{array}{c}
-0.036963 \\
-0.003304 \\
0.053801
\end{array}\right), \gamma_{4}^{\prime}=\left(\begin{array}{c}
0.952770 \\
-0.351912 \\
-0.053691
\end{array}\right)^{T}, \gamma_{5}^{\prime}=\left(\begin{array}{c}
-0.036943 \\
-0.003302 \\
0.053772
\end{array}\right) .
\end{gathered}
$$

In Fig. 7, we show the bifurcation diagrams by using direct numerical simulation (Fig. 7(a))and the local mapping (Fig. 7(b)) for the rigid model. It is shown that the stable periodic motion evolves into chaotic motion through grazing bifurcation, and the bifurcation diagram given by the local mapping method compares well with that given by direct numerical simulation method.

\section{Conclusions}

In this paper, the double grazing bifurcations of two classes of non-smooth railway wheelset models are investigated. The non-smoothness at the railway wheelset systems mainly come 


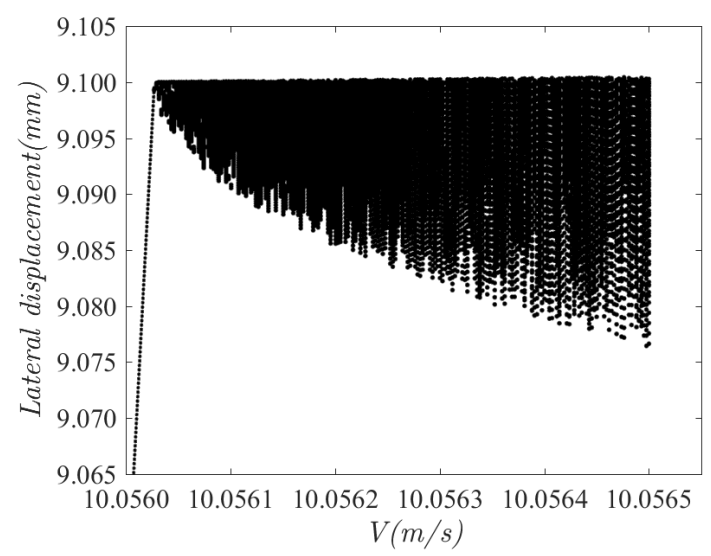

(a) Numerical simulation,

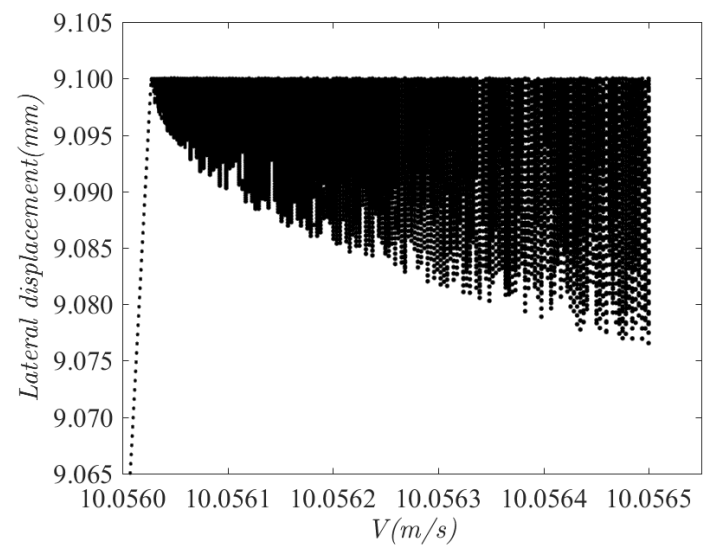

(b) The PDM

Figure 7: Bifurcation diagrams for the rigid impact model .

from flange impact. We consider two types of flange impact models. To understand and explain the dynamical behavior of the railway wheelset systems caused by the grazing bifurcations, the PDM approach is used to construct the Poincaré maps of the systems near grazing orbit. Numerical simulation results indicate that the Poincaré-section discontinuity mapping approach is valid for the railway wheelset system with both rigid and soft impact.

It is shown that the chaotic motion appears after gazing bifurcation in the rigid impact model of the railway wheelset system, which corresponds to one of the scenarios described in Nordmark [17]. In the soft impact model with symmetric bilateral constraints, the system has a pitchfork bifurcation and tends to chaos through period doubling after grazing bifurcation. Such phenomenon is different from that of unilateral constraint system. In unilateral constraint system, it translates directly to chaos through period doubling. We consider one parameter grazing bifurcation of the railway wheelset in this work. The degenerate grazing bifurcation of the system will be considered in our future work.

\section{Acknowledgments}

This work is supported by the National Natural Science Foundation of China (Nos. 12072291, 12172306 and 11732014).

\section{Conflict of Interest}

The authors declare that they have no conflict of interest. 
Table 1: Values of parameters [5]

\begin{tabular}{ll}
\hline Parameter & Value \\
\hline Mass of the wheelaxle & $m=1022 \mathrm{~kg}$ \\
Moment of inertia & $I=678 \mathrm{kgm}^{2}$ \\
Half of the track gauge & $a=0.716 \mathrm{~m}$ \\
Distance from centre of gravity to $k_{2}$ & $d_{1}=0.620 \mathrm{~m}$ \\
Shear modulus & $G=808 \mathrm{MNm}^{-2}$ \\
Major semiaxis of contact ellipse & $a_{e}=6.578 \mathrm{~mm}$ \\
Minor semiaxis of contact ellipse & $b_{e}=3.937 \mathrm{~mm}$ \\
Dead band & $\delta=9.1 \mathrm{~mm}$ \\
Spring constant & $k_{0}=14.6 \mathrm{MNm}^{-1}$ \\
Lateral stiffness of primary suspension & $k_{1}=1.823 e 4 \mathrm{Nm}^{-1}$ \\
Yaw spring stiffness of primary suspension & $k_{2}=0 \mathrm{Nm}^{-1}$ \\
Lateral wheel-rail contact parameter & $\Psi=0.54219$ \\
Longitudinal wheel-rail contact parameter & $\Phi=0.60252$ \\
Centred wheel rolling radius & $r_{0}=0.4572 \mathrm{~m}$ \\
Wheel conicity & $\lambda=0.05$ \\
Coefficient of friction & $\mu=0.15$ \\
N is the vertical force between wheel and rail & $\mu N=10 \mathrm{KN}$ \\
Running velocity & $V-$ \\
\hline
\end{tabular}

\section{Data Availability Statements}

The datasets generated during and/or analysed during the current study are available from the corresponding author on reasonable request.

\section{Appendix. Calculating the PDM of the soft impact model}

Suppose that a dynamical system discontinuity can be represented by state space discontinuity surface $D$, and that the system has a single discontinuity boundary $\Sigma=\{X \in D \mid H(X, V)=0\}$. Consider the $\mathrm{n}$ degree of freedom soft impact autonomous system

$$
\dot{X}= \begin{cases}F_{1}(X, V), & H(X, V)<0 \\ F_{2}(X, V), & H(X, V) \geq 0\end{cases}
$$

where $X \in \mathbb{R}^{2 n}, V \in \mathbb{R}, F_{1}, F_{2}: \mathbb{R}^{2 n} \longmapsto \mathbb{R}^{2 n}$ are supposed to be sufficiently smooth. The flows $\phi_{i}(X, V, t),(i=1,2)$ generated by each of the vector fields can be defined as the quantities that satisfy

$$
\frac{\partial \phi_{i}(X, V, t)}{\partial t}=F_{i}\left(\phi_{i}(X, V, t)\right), \phi_{i}(X, V, 0)=X .
$$

Expanding each of the system flows $\phi_{i}(X, V, t)$ in a Taylor series about the grazing point $\left(X^{*}, V^{*}, 0\right)$, we obtain

$$
\begin{aligned}
\phi_{i}(X, V, t) & =X^{*}+\left[\left(X-X^{*}\right)+F_{i}^{*} t\right]+\left[\frac{1}{2} F_{i, X}^{*} F_{i}^{*} t^{2}+F_{i, X}^{*}\left(X-X^{*}\right) t+F_{i, V}^{*}\left(V-V^{*}\right) t\right]+\text { h.o.t } \\
& =X+F_{i}^{*} t+\left[\frac{1}{2} F_{i, X}^{*} F^{*} t^{2}+F_{i, X}^{*}\left(X-X^{*}\right) t+F_{i, V}^{*}\left(V-V^{*}\right) t\right]+\text { h.o.t }
\end{aligned}
$$


where $F_{i}^{*}=F_{i}\left(X^{*}, V^{*}\right), F_{i, X}^{*}=\left.\frac{\partial F_{i}(X, V)}{\partial X}\right|_{X=X^{*}, V=V^{*}, F_{i, V}^{*}}=\left.\frac{\partial F_{i}(X, V)}{\partial V}\right|_{X=X^{*}, V=V^{*}}(i=1,2)$.

We define the Poincaré section as

$$
\Pi_{N}=\left\{X \in D \mid v=\frac{\partial H(X, V)}{\partial t}=0\right\} .
$$

Suppose that the state point $X_{0} \in \Pi_{N}$ represents the initial point in the neighborhood of grazing point $X^{*}$. In order to construct the Poincaré-section discontinuity mapping (PDM), we evolve the flow $\phi_{1}$ backward starting from point $X_{0}$ until it intersects the discontinuity boundary $\Sigma$ at point $X_{1}$. The time it takes from $X_{0}$ to $X_{1}$ is denoted by $-\delta_{1}$. The forward evolution using $\phi_{2}$ by a time $\delta_{2}$ until it intersects $\Sigma$ again at point $X_{2}$. Finally, using $\phi_{1}$ backwards until it reaches the Poincaré section $\Pi_{N}$ again at point $X_{3}$ after a time $\delta_{3}$. The PDM is the map $X_{0} \mapsto X_{3}$, and it has the form:

$$
\operatorname{PDM}\left(X_{0}, V\right)= \begin{cases}X_{0}, & H\left(X_{0}, V\right)<0 \\ \phi_{1}\left(\phi_{2}\left(\phi_{1}\left(X_{0}, V, \delta_{1}\right), V, \delta_{2}\right), V,-\delta_{3}\right), & H\left(X_{0}, V\right) \geq 0 .\end{cases}
$$

We then calculate PDM in the following three steps,

Step1. From $X_{0}$ to $X_{1}$.

According to Eq. (5.3), we have

$$
\begin{aligned}
X_{1} & =\phi_{1}\left(X_{0},-\delta_{1}, V\right) \\
& =X_{0}-F_{1}^{*} \delta_{1}+\frac{1}{2} F_{1, X}^{*} F_{1}^{*} \delta_{1}^{2}-F_{1, X}^{*}\left(X_{0}-X^{*}\right) \delta_{1}-F_{1, V}^{*}\left(V-V^{*}\right) \delta_{1}+\text { h.o.t. }
\end{aligned}
$$

Since the point $X_{1}$ is on $\Sigma$, we have $H\left(X_{1}, V\right)=0$. Expanding $H\left(X_{1}, V\right)$ with respect to $\delta_{1}$, we have

$$
\begin{aligned}
H\left(X_{1}, V\right) & =H\left(\phi_{1}\left(X_{0},-\delta_{1}, V\right), V\right) \\
& =H\left(X_{0}, V\right)-v_{1}\left(X_{0}, V\right) \delta_{1}+\frac{1}{2} a_{1}\left(X_{0}, V\right) \delta_{1}^{2}+\text { h.o.t } \\
& =0,
\end{aligned}
$$

where $v_{i}(X, V)=\frac{\partial H(X, V)}{\partial t}=H_{X} F_{i}$ is the generalized velocity and $a_{i}(X, V)=\frac{\partial^{2} H(X, V)}{\partial t^{2}}=$ $H_{X} F_{i, X} F_{i}$ is generalized acceleration $(i=1,2)$.

By Eq. (5.7) and that $v_{1}\left(X_{0}, V\right)=0$, we have

$$
\delta_{1}=\sqrt{\frac{-2 H\left(X_{0}, V\right)}{a_{1}^{*}}}+\text { h.o.t. }
$$

Step2. From $X_{1}$ to $X_{2}$.

According to Eq. (5.3), we have

$$
\begin{aligned}
X_{2} & =\phi_{2}\left(X_{1}, \delta_{2}, V\right) \\
& =X_{1}+F_{2}^{*} \delta_{2}+\frac{1}{2} F_{2, X}^{*} F_{2}^{*} \delta_{2}^{2}+F_{2, X}^{*}\left(X_{1}-X^{*}\right) \delta_{2}+F_{2, V}^{*}\left(V-V^{*}\right) \delta_{2}+\text { h.o.t. }
\end{aligned}
$$


Since the point $X_{2}$ is in the surface $\Sigma$, we have $H\left(X_{2}, V\right)=0$. Expanding $H\left(X_{2}, V\right)$ with respect to $\delta_{2}$, we have

$$
\begin{aligned}
H\left(X_{2}, V\right) & =H\left(\phi_{2}\left(X_{1}, \delta_{2}, V\right), V\right) \\
& =H\left(X_{1}, V\right)+v_{2}\left(X_{1}, V\right) \delta_{2}+\frac{1}{2} a_{2}\left(X_{1}, V\right) \delta_{2}^{2}+\text { h.o.t } \\
& =0 .
\end{aligned}
$$

According to Eq. (5.10) and $H\left(X_{1}, V\right)=0$, we have

$$
\begin{aligned}
\delta_{2} & =\frac{-2 * v\left(X_{1}, V\right)}{a_{2}^{*}}+\text { h.o.t } \\
& =\frac{2}{a_{2}^{*}}\left[a_{1}^{*}+a_{1, X}^{*}\left(X_{0}-X^{*}\right)+a_{1, V}^{*}\left(V-V^{*}\right)\right] \delta_{1}+\text { h.o.t. }
\end{aligned}
$$

Step3. From $X_{2}$ to $X_{3}$.

According to Eq. (5.3), we have

$$
\begin{aligned}
X_{3} & =\phi_{1}\left(X_{2},-\delta_{3}, V\right) \\
& =X_{2}-F_{1}^{*} \delta_{3}+\frac{1}{2} F_{1, X}^{*} F_{1}^{*} \delta_{3}^{2}-F_{1, X}^{*}\left(X_{2}-X^{*}\right) \delta_{3}-F_{1, V}^{*}\left(V-V^{*}\right) \delta_{3}+\text { h.o.t. }
\end{aligned}
$$

Since the point $X_{3}$ is on the Poincaré section $\Pi_{N}$, it means $v_{1}\left(X_{3}, V\right)=0$. Expanding $v_{1}\left(X_{3}, V\right)$ with respect to the grazing point $\left(X^{*}, V^{*}, 0\right)$, and according to Eqs.(5.6)) and (5.9) we obtain

$$
\begin{aligned}
v_{1}\left(X_{3}, V\right) & =v_{1}\left(\phi\left(X_{2},-\delta_{3}, V\right), V\right) \\
& =\frac{1}{2} a_{1, X}^{*} F_{1}^{*} \delta_{1}^{2}+\frac{1}{2} a_{2, X} F_{2}^{*} \delta_{2}^{2}-\left[a_{1}^{*}+a_{1, X}^{*}\left(X_{0}-X^{*}\right)+a_{1, V}^{*}\left(V-V^{*}\right)\right] \delta_{1} \\
& +\left[a_{2}^{*}+a_{2, X}^{*}\left(X_{0}-X^{*}\right)+a_{2, V}^{*}\left(V-V^{*}\right)-a_{1, X}^{*} F_{1}^{*} \delta_{1}\right] \delta_{2}-\left[a_{1}^{*}+a_{1, X}^{*}\left(X_{0}-X^{*}\right)(5 .\right. \\
& \left.+a_{1, V}^{*}\left(V-V^{*}\right)-a_{1, X}^{*} F_{1}^{*} \delta_{1}+a_{2, X}^{*} F_{2}^{*} \delta_{2}\right] \delta_{3}+\frac{1}{2} a_{1, X}^{*} F_{1}^{*} \delta_{3}^{2}+\text { h.o.t } \\
& =0 .
\end{aligned}
$$

Suppose $\delta_{3}$ has the form:

$$
\begin{aligned}
\delta_{3} & =\alpha_{1} \delta_{1}+\alpha_{2} \delta_{2}+\alpha_{3}\left(X_{0}-X^{*}\right) \delta_{1}+\alpha_{4}\left(V-V^{*}\right) \delta_{1}+\alpha_{5}\left(X_{0}-X^{*}\right) \delta_{2} \\
& +\alpha_{6}\left(V-V^{*}\right) \delta_{2}+\alpha_{7} \delta_{1} \delta_{2}+\alpha_{8} \delta_{1}^{2}+\alpha_{9} \delta_{2}^{2}+\text { h.o.t. }
\end{aligned}
$$

Substituting Eq.(5.15) into Eq.(5.13) and comparing the coefficients of the same terms, yields $\alpha_{1}=-1, \alpha_{2}=1, \alpha_{3}=\alpha_{4}=\alpha_{8}=0, \alpha_{5}=\frac{a_{2, X}^{*}-a_{1, X}^{*}}{a_{1}^{*}}, \alpha_{6}=\frac{a_{2, V}^{*}-a_{1, V}^{*}}{a_{1}^{*}}, \alpha_{7}=\frac{1}{a_{1}^{*}}\left(a_{2, X}^{*} F_{2}^{*}-\right.$ $\left.a_{1, X}^{*} F_{1}^{*}\right), \alpha_{9}=\frac{1}{2 a_{1}^{*}}\left(a_{1, X}^{*} F_{1}^{*}-a_{2, X}^{*} F_{2}^{*}\right)$.

Substituting Eqs.(5.8), (5.11), (5.15), (5.6) and (5.9) into (5.12), we obtain

$$
\begin{aligned}
X_{3} & =X_{0}+2\left[\left(F_{2, X}^{*}-F_{1, X}^{*}\right)+\frac{F_{1}^{*}\left(a_{1, X}^{*}-a_{2, X}^{*}\right)}{a_{1}^{*}}\right]\left(X_{0}-X^{*}\right) \sqrt{\frac{-2 H\left(X_{0}, V\right)}{a_{1}^{*}}} \\
& +2\left[\left(F_{2, V}^{*}-F_{1, V}^{*}\right)+\frac{F_{1}^{*}\left(a_{1, V}^{*}-a_{2, V}^{*}\right)}{a_{1}^{*}}\right](V-V *) \sqrt{\frac{-2 H\left(X_{0}, V\right)}{a_{1}^{*}}}+\text { h.o.t. }
\end{aligned}
$$


So, the expression of $\operatorname{PDM}\left(X_{0}, V\right)$ is

$$
\begin{cases}X_{0}, & H\left(X_{0}, V\right)<0, \\ X_{0}+2\left[\left(F_{2, X}^{*}-F_{1, X}^{*}\right)+\frac{F^{*}\left(a_{1, X}^{*}-a_{2, X}^{*}\right)}{a_{1}^{*}}\right]\left(X_{0}-X^{*}\right) \sqrt{\frac{-2 H\left(X_{0}, V\right)}{a_{1}^{*}}}, & \\ +2\left[\left(F_{2, V}^{*}-F_{1, V}^{*}\right)+\frac{F^{*}\left(a_{1, V}^{*}-a_{2, V}^{*}\right)}{a_{1}^{*}}\right](V-V *) \sqrt{\frac{-2 H\left(X_{0}, V\right)}{a_{1}^{*}}+h . o . t,} & H\left(X_{0}, V\right) \geq 0 .\end{cases}
$$

\section{References}

[1] P. G. Thomsen, H. True, Non-smooth Problems in Vehicle Systems Dynamics, Proc. Euromech 500 Colloquium, Springer, Berlin, Wien, New York, 2010.

[2] R. R. Huilgol, Hopf-Friedrichs bifurcation and the hunting of a railway axle. Quart. Appl. Math. 1978, 36: 85-94.

[3] C. Kaas-Petersen, Chaos in a railway bogie, Acta Mechanica, 1986, 61: 89-107.

[4] J. P. Meijaard, A. D. De Pater, Railway vehicle systems dynamics and chaotic vibrations, Int. J. Non-Linear Mech., 1988, 24: 1-17.

[5] C. Knudsen, R. Feldberg, H. True, Bifurcations and chaos in a model of a rolling railway wheel set. Philos Trans R. Soc. Lond A, 1992, 338: 455-69.

[6] C. Knudsen, E. Slivsgaard, M. Rose, H. Ture, R. Feldberg, Dynamics of a model of a railway wheelset. Nonlinear Dyn., 1994, 6(2): 215-236.

[7] H. True, Dynamics of a rolling wheelset. Appl. Mech. Rev., 1993, 46: 438-444.

[8] M. Ahmadian, S. P. Yang, Hopf bifurcation and hunting behaviour in a rail wheelset with flange contact. Nonlinear Dyn., 1998, 15: 15-30.

[9] M. Ahmadian, S. P. Yang, Effect of system nonlinearities on locomotive bogie hunting stability. Veh. Syst. Dyn., 1998, 29(6): 365-384.

[10] H. True, R. Asmund, The dynamics of a railway freight wagon wheelset with dry friction damping. Veh. Syst. Dyn., 2002, 38: 149-163.

[11] M. Hoffmann, H. True. Dynamics of two-axle railway freight wagons with UIC standard suspension. Veh. Syst. Dyn., 2006, 44: 139-146.

[12] M. Hoffmann. On the dynamics of European two-axle railway freight wagons. Nonlinear Dyn., 2008, 52(4): 301-311.

[13] X. J. Gao, Y. H. Li, Y. Yuan et al., Symmetric/asymmetric bifurcation behaviours of a bogie system. J. Sound. Vib., 2013, 332(4): 936-951.

[14] H. True, A. P. Engsig-Karup, D. Bigoni, On the numerical and computational aspects of non-smoothnesses that occur in railway vehicle dynamics. Math. Comput. Simul., 2014, 95: 78-97.

[15] R. I. Leine, H. Nijmeijer. Dynamics and bifurcations of non-smooth mechanical systems. Springer, New York, 2004.

[16] M. di Bernardo, C. J. Budd, A. R. Champneys, et al., Piecewise-smooth Dynamical Systems: Theory and Applications. Springer, London, 2008.

[17] A. B. Nordmark, Non-periodic motion caused by grazing incidence in an impact oscillator. J. Sound Vib., 1991, 145: 279-297.

[18] W. Chin, E. Ott, H. E. Nusse, C. Grebogi, Grazing bifurcations in impact oscillators. Phys. Rev. E., 1994, 50: $4427-4444$.

[19] W. Chin, E. Ott, H. E. Nusse, C. Grebogi, Universal behavior of impact oscillators near grazing incidence. Phys. Lett. A, 1995, 201:1 97-204.

[20] D. H. Li, H. B. Chen, J. H. Xie, et al., Sinai-Ruelle-Bowen measure for normal form map of grazing bifurcations of impact oscillators. J. Phys. A, 2017, 50: 386103.

[21] P. C. Miao, D. H. Li, Y. Yue et al., Chaotic attractor of the normal form map for grazing bifurcations of impact oscillators. Physica D, 2019, 398: 164-170.

[22] M. H. Fredriksson, A. B. Nordmark, Bifurcations caused by grazing incidence in many degrees of freedom impact oscillators. Proc. R. Soc. A, 1997, 453: 1261-1276. 
[23] M. di Bernardo, C. J. Budd, A. R. Champneys, Normal form maps for grazing bifurcations in n-dimensional piecewise-smooth dynamical systems. Physica D, 2001, 160(3-4): 222-254.

[24] Q. Li, L. Wei, J. Tan, et al. Double Grazing Periodic Motions and Bifurcations in a Vibroimpact System with Bilateral Stops. Abstr. Appl. Anal., 2014, 2014:1-9.

[25] S. Yin, G. L. Wen, H. D. Xu, et al., Higher order zero time discontinuity mapping for analysis of degenerate grazing bifurcations of impacting oscillators. J. Sound. Vib., 2018, 437: 209-22.

[26] J. Ing, E. Pavlovskaia, M. Wiercigroch, et al., Bifurcation analysis of an impact oscillator with a one-sided elastic constraint near grazing, Physica D, 2010, 239: 312-321.

[27] H. Dankowicz, X. P. Zhao, Local analysis of co-dimensionone and co-dimension-two grazing bifurcations in impact microactuators. Physica D, 2005, 202: 238-257.

[28] H. B. Jiang, A. S. E. Chong, Y. Ueda, et al., Grazing-induced bifurcations in impact oscillators with elastic and rigid constraints, Int. J. Mech. Sci., 2017,127: 204-214.

[29] P. J. Vermeulen, K. L. Johnson. Contact of nonspherical elastic bodies transmitting tangential forces. J. Appl. Mech., 1964, 31:338-40. 